\title{
Index
}

27 horas 9,137

$A B C 4,35,56,75,81,143,146$

Aborto criminal 74

Adiós, cigüeña, adiós 81

Aguirresarobe, Javier 137n13

alarms $21,68,69$, 73, 88, 96, 98, $155,172 \mathrm{n} 11$

Alianza Popular 59, 71-72, 97, 102n

Amnesty Laws 80, 84, 162

amor brujo, El 186

Andalucia 2, 43, 177

Andreu, Simón 59

Aranda, Vicente 9, 27

Arias Aranzueque, Jesús ('el Susi')

145-147, 188

Armendáriz, Montxo 9, 137

Asignatura pendiente 91

Attali, Jacques 16

Balañá, Pedro 167

Bandrés, Juan María 121-122, $137 \mathrm{n} 10$

Barthes, Roland 144

Basque Country see Euskadi

Bernaola, Carmelo 91

Bilbao 119-120, 122

Blaxploitation 10, 24n10

boda del señor cura, La 68
Bodas de sangre 186

Bodegas, Roberto 90, 207n12

Boney M. 2, 23n1

Bonnie and Clyde 88

Bosch, Joan 27

Brigada central 9

Burning 115, 148

Cabal, Fermín 129

Cabré, Berta 59

caló 141

Campo de la Bota 23n5, 43, 44, 45, 65n18, 170, 195-198

Camus, Mario 5

Caño Roto (neighbourhood in

Madrid) 77, 176-177,

206n1

caño roto (musical genre) 77, 176-177, 203

Carmen 186

Carretero, Gil 8, 68

cars

car chases 28, 32, 34, 35, 39, 45, $47,49,50,57,63 \mathrm{n} 5,84$, 164, 184, 192

car stunts $28,39,60$

car theft 1, 20, 26, 32, 34-36, 38, $44,59,108,139,150,187$, 188,51 
SEAT 124 1, 26, 32, 37-38, 47, 51

SEAT 600 37-38

SEAT factory 61-62

cassette tapes see radio cassettes

Celda 21141

censorship 6, 11, 34, 36, 39, 73, 76, 120, 178

Centro de Investigaciones

Sociológicas 70

Cercas, Javier 17

cheli 143-144, 169, 171n2

Chion, Michel 20, 96

Chocolate 8, 68

Christiane F. 12

cines de barrio 10, 27, 65n20

cine de destape 7, 18, 76, 81, 184

cine neoquinqui (or neokinki) 208

civil insecurity see public order

Cohen, Stanley 75, 100

Colegas 8, 103

comedia madrileña 8

Constitution of 1978 14, 21, 64n11, 67, 70, 72, 79, 94, 101n1, 155, 162

COPEL 54, 163

co-productions 28, 109, 129 , 144-145

Córdoba, Juan Vicente 177, 208

Coto de caza 81

Criando ratas 208

crime

anti-theft systems 69-70

bank robberies $36,56-58,82-84$, $99,133,146-147$

burglary 3-4, 111, 117

car theft see cars, car theft

crime cinema 9

crime rates $3-4,21,23,35,40$, 67

crime survey $70-71,102 \mathrm{n} 2$

crime waves 4,69 public anxieties surrounding 4 , $21,34,35,40,51,67-68$, 74, 76-77, 81, 96-101

underage crime $26,30,40-41$, 64n15, 76, 79

crimen de Cuenca, El 102n4, 120

de la Iglesia, Eloy 8, 12, 19, 23n6, 89-101, 103-138

relationship with José Luis

Manzano 108-109,

135-136

de la Loma, José Antonio 5-8, 23n5, 26-66, 156-173

Delgado, Juan Carlos ('el Pera') $63 n 5$

Del rosa al amarillo 80

Deprisa, deprisa 8, 18, 23n7, 49, 55, 81, 139-155, 171n4, 185-194, 200-202

production of 145-146

exhibition of 147-149

reception of 148

desarrollismo 2-3, 21, 36-37, 191

detention centres see reformatories

Diario 16 95, 121-122, 162

Dibildos, José Luis 90

diputado, El 8, 89, 109

direct sound 22, 139, 140, 144, 151-152, 155, 156-157, $172 \mathrm{n} 9$

droga es joven, La 68

drugs

cannabis $171 \mathrm{n} 2,108,184,194$

drug dealing 53, 108, 141

drug trafficking 119, 123-124,

128-129, 132

and ETA 121, 132

heroin $9,22,53,103-104,112$,

118-137

and police repression 118-123 
dubbing $86,99,109,112-113$, 137n6, 151-152, 154 post-synch sound 113,152, 156, $172 \mathrm{n} 9$

Dulces navajas 109

'economic miracle' see desarrollismo El Caso 2, 9, 38, 40, 68, 107, 136n $3,147,149$, 203

El Coleta see Ramsés Gallego

El Imparcial 4, 75, 93

E1 Jaro see José Joaquín Sánchez Frutos

E1 Lute see Eleuterio Sánchez

El Lute: Camina or revienta 9-10, $24 \mathrm{n} 8$

El Lute 2: Mañana seré libre 9

El País 29, 144

El Pera see Juan Carlos Delgado

E1 Pirri see José Luis Fernández Eguia

E1 Torete see Ángel Fernández Franco

E1 Vaquilla see Juan José Moreno Cuenca

Emanuelle y Carol 73

erotic cinema 51, 59, 73-80, 89, 93, $101,102 \mathrm{n} 4,113$

espontáneo, El 5

estanquera de Vallecas, El 8, 23n6, 103, 135

Estopa 181

ETA 65 n15, 71, 91, 121, 132-134, $137 \mathrm{n} 13,168$

Euskadi 2, 9, 104, 118-135, $137 \mathrm{n} 13,167$

exhibition of cine quinqui 9-10, 13-14, 27, 46, 81, 156, $172 \mathrm{n} 8$

Extremadura 2, 177
Fassbinder, Rainer Werner 105

Fernández Eguia, José Luis ('el Pirri') 8, 49, 108-109, 129, 131, 138n17

Fernández Franco, Ángel ('el Torete') 5-7, 20-66, 156, 158, 182-185

Fernández Franco, Basilio 30, 48

Ferrandis, Antonio 90, 99

Ferreri, Marco 5, 89

Films Moliere 144

Films Zodíaco 34

Flores de luna 177

Forqué, José María 89

Fotogramas 29, 50, 65n23

Fraga, Manuel 71, 197

Franco, Francisco 2, 4, 14, 93, 154, 162,168

death of 4,14

Franco regime 2, 3, 6, 14, 23n1, $29,39,40,42,68,70,76$, $80,90,124,144,155,158$, 174

authoritarianism of the Franco regime $4,7,14,60,70,93$, $124,155,171,210$ nostalgia for the Franco regime 80,93

FRAP 79-80, 84-85

Fuerzas de Orden Público 70, 79

GAL 134

Gallego, Ramsés ('el Coleta') 175, 199-206, 208

gambita 101

gangster film 10, 140

Garci, José Luis 90-91

Guardia Civil see police

Guillén, Fernando 57, 129

Gutiérrez Aragón, Manuel 8, 153 
gypsies 1, 2, 15, 22, 44-45, 141, $157,168,174,175-178$, 184, 193

Hebdige, Dick 16

heroin see drugs

Hijos de papá 68

Historia de 'S' 80

homoeroticism 103, 122, 126

housing

overcrowding 36

shanty towns $32,43,55,65 \mathrm{n} 18$, $143,179,184,196-97$, 206n1

tower blocks 1-2, 32, 35, 43-45, 201, 204

urban planning 179, 206n1

UVAs 2-3

IFI 73-74

Interviú 8, 52, 143, 147, 167-68

Iquino, Ignacio F. 7-8, 23n5, 27, $28,62,67,73-79$

Iriondo, Luis 122

Isas-Isasmendi, Antonio 27, 62

jardin de las delicias, El 52

Jarfaiter 203-204

Jubilee 12

Jugando con la muerte 28

Julienne, Rémy 28, 56, 61-62

juvenile detention centres see reformatories

Kalender Films 80-81

Katz, Jack 11, 116

Klimovsky, León 7-8, 68, 74

Kubrick, Stanley 11, 115

La Luna de Madrid 144

La Marelu 185
La Mina 5, 29, 30-36, 43-45, 58-59, 64n6, 65n18, 74, 156, 168

LaBelle, Brandon 161, 188-90, 196, 199, 209-210

Lara Polop, Francisco 67, 80, $82-83,87,89$

Las Grecas 176-177, 202-203

Lefebvre, Henri 44, 54

Ley de Orden Público 101n1

Ley de Protección de Menores $64 \mathrm{n} 15$

Ley, Linda 74, 77-78

Ley de Amnistía 80, 84, 162

Ley General de Educación 3, 76, $102 \mathrm{n} 6$

leyes de la frontera, Las 17

location shooting $5,8,23 \mathrm{n} 5,26-27$, 29, 64n6, 74-75, 77, 89, $131,139,149,156$

Lole y Manuel 185-186, 193

Los Chichos 15, 98, 115, 164, 174, 177-181, 187, 194-199

Los Chorbos 176-177

Los Chunguitos 15, 22, 98, 174, 178, 180, 182-194, 200, 205-206

M-30 motorway $84,179,187$, 200-201

magnifico Tony Carrera, El 28

Mamá cumple cien años 145

Manzano, José Luis 8-9, 19, 21, 103-138, 203-204

death of 136 discovery of 108

Maravillas 8, 153

Marks, Laura 111-112, 114, 122-123

Massumi, Brian 17

Matar al Nani 207n12 
Mattei, Danilo 79, 83, 87

Menz, Bernardo 152, 155

Mercero, Antonio 9

mercheros see quinquilleros

Metralleta Stein 28, 63n6

microphone 61, 139, 152

Miedo a salir de noche 8, 67-68, 73, 89-101, 105, 107, 124

exhibition of 89

production of 89

migration $2,3,37,43,77,143,175$, 177-179

'miracle years' see desarrollismo

Miró, Pilar 23n7, 81, 102n4, 120 ley Miró 120, 137n9

Molins, Marta 123

Moncloa Pacts, the 14, 24n14, 85

Montllor, Ovidi 123

moral panics 4, 7-8, 22, 33, 100, 110

Moreno Cuenca, Juan José ('el Vaquilla') 5, 7, 18, 22, 30-33, 41, 47, 58, 62-63, 66n27-28, 156-173

muerte de Mikel, La 137n13 music videos 195, 201-204

Navajeros 8, 12, 16, 41, 103-118, $122,137 \mathrm{n} 7$

production of 108-109

exhibition of 109-110

niña de luto, La 184

novios búlgaros, Los 136

Nuevo Cine Español 80

nuevos españoles, Los 90

ojos vendados, Los 145

Ozores, Mariano 76

paella westerns 18, 74

Party 129, 137n15

Pasolini, Pier Paolo 105 patria de 'el Rata', La 8, 21, 67, 68, 79-89, 102n9

exhibition of 82

production of 81

reception of 82

PCE (Partido Comunista de

España) 8, 85, 102n10, $136 n 2$

Penal Code 14, 162

penal system 131-132, 166, 171

Penitentiary Law 163, 172n16

Peret 175, 177, 180

Pérez Giner, José Antonio 34, $137 \mathrm{n} 9$

Perras callejeras 7

Perros callejeros 5-7, 12-13, 24n8, 26-46, 59, 65n20, 74, 79, $107,110,137 \mathrm{n} 9,156$

casting of $30-31$

censorship of 34

exhibition of 12-13, 24n8, 34, 46

reception of 35

Perros callejeros 2: busca y captura 7,

$9,20,26,46-55,182-185$

reception of 47

exhibition of 47

Petit, Victor 39

pico, El 8, 10, 24n8-9, 34, 49, 103-104, 112, 118-128, 134-135

production of $120-121$

pico 2, El 8, 10, 14, 21, 34, 103-

$104,112,118,128-135$,

138n16, 203-204

exhibition of 131

Pixote 12

placeres ocultos 8, 109

police

Guardia Civil 30, 39, 45, 119-20, 131, 164

Policía Armada 172n10

Policía Nacional 172n10 
police brutality 1, 6-7, 158, 185, 209

police reform $70,172 \mathrm{n} 10$

post-synch sound see dubbing Pozo del Tío Raimundo 143, 177

prisons

La Modelo 53-55, 164, 209

Ocaña 156, 158, 160, 173n20, 198, 209

penitentiary reform 14,162 prison escapes $54,163,165$

prison mutinies $41,54,162-166$, $173 \mathrm{n} 20$

prisoners

political prisoners 80 , 162-163

presos sociales $162-163$

soundscape of 160-162

PSOE 4, 71, 85, 119, 121, 134

public order 21, 67-68, 70-73, 75-76, 78, 89, 92, 97, 101-102n1

\section{Quadrophenia 12}

quatre cents coups, Les 164

Querejeta, Elías 144-146, 163

Quinlivan, Davina 114

Quinqui Stars 208-209

quinquilleros 1, 18, 142

origin of term 1

radio cassettes $15,22,77,174$, 180-181, 186-188, 194195, 204-205

rap quinqui 175, 199-206

rape $54,75-79,93-95,111,114$

recession 3, 24n14, 176, 200, 208

redada, La 23n 5, 28

reformatories 1, 5, 12, 30-31, 40-43, 79, 158, 160-162, $172 \mathrm{n} 13$ reina del mate, La 129

rock urbano 115

Rosalía 206

Rumba Tres 115

rumbas $15,98,132,174-207$

rumba catalana 175-176

rumba vallecana 177-181, 195, 200

'S'-rated films 73, 76, 79-80, 89, $93,102 n 4$

Sabina, Joaquín 107

sacerdote, El 101

Sacristán, José 89, 91-94, 99, 105 89-90 101

sainete 89-90, 101

Sáinz, Tina 95, 137n6

Salvador, Julio 23n5, 29

San Francisco, Enrique 111, 125-126, 208

San Sebastián 9, 120, 122, $137 \mathrm{n} 13$

Sánchez Frutos, José Joaquín ('el Jaro') 8, 41, 103-105, 107

Sánchez, Eleuterio ('el Lute') 2, 9-10, 23n1, 107, 178

Saura, Carlos 5, 8, 139, 145-152, 155-56, 163, 172n9, 186

schools 3, 59, 76-77

Seray, Bernard 47, 56, 58, 74, 78, 208

shanty towns see housing, shanty towns

silence $82,155,160-161,185,194$, 209

Sin la sonrisa de Dios 29

slang 1, 5, 14, 16, 22, 35, 109, $119,139,140-144$, 169-170

Socuéllamos, Berta 145, 148, 192-193 
sound effects 15,127

soundscapes

and social order 155

of cinema auditoria 1, 12-15, $34,46,82,109,131$, 146

of the car $187-190$

of the home 72-73, 96-101

of prisons see prisons, soundscape of

urban soundscapes 187, 189 soundtrack albums 15, 22, 174 , 182-183, 185, 194, 206

Spanish Civil War 97, 137n11, 154

Suárez, Adolfo 86, 90, 162

Summers, Manuel 80-81, 102n9

tabloid press 2, 11, 40, 69, 74, 88-89, 91, 129, 131, 135

\section{Taxi 186}

Tejero, Antonio 120, 125

tercera vía 81, 90-91, 101

terrorism 4, 71-73, 79-80, 84, 89-90, 92, 98, 119, 121, 123-124, 129, 133-134, 163

The Gypsy Kings 186

Thompson, Marie 14-15

Tomlinson, John 37, 39

Torres, Maruja 50-51, 63n4 torture 122, 139, 162, 165, 209

Tribunal de Orden Público 70

Tribunales Tutelares de Menores 42

Triunfo 104, 162

Trueba, Fernando 29, 35, 44, 63n3

Turno de oficio 9

UCD (Unión de Centro

Democrático) 71-72, 79, 85-86, 90
Ugal Cuenca, Miguel ('el Carica') $30,42,47,63,65 \mathrm{n} 22$

último sábado, El 5

uiltimos golpes de 'el Torete', Los 7, 20, 26, 55-62

reception of 55

Umbral, Francisco 144, 169-170

Una gota de sangre para morir amando 12,115

unemployment $3,12,24 n 14,67,71$, 80, 85, 108, 208

Unidades Vecinales de Absorción (UVAS) see housing

Valdelomar, José Antonio ('el

Mini') 49, 81, 145-150, $171 \mathrm{n} 4,172 \mathrm{n} 5$

Vallecas 8, 108, 115, 143, 177-180, 187, 193, 200

rumba vallecana see rumbas, rumba vallecana

victimisation $67,68,71,73$

videos comunitarios 10

Vigil, Luis Martín 68

Villaverde 82, 145, 186-187

Vinader, Xavier 166-168

vinyl records 176, 204

violadores del amanecer, Los 7, 21, 67-68, 73-75, 78-79, 86, 93, 101

reception of 75

Vizcaíno Casas, Fernando 68 voice $1,9,22,35,46,53,59,76,86$, $99,109,112,139-172,178$, 209

dubbing see dubbing vocal performance $19,46,113$, 140, 149-156, 159-160, 170

Volando voy $63 \mathrm{n} 5$

Warriors, The 12-13 
Yo, 'el Vaquilla' 7, 14, 22, 139-140, 144, 156-171, 173n20 production of 168 reception of 156-157

Young Sánchez 5 youth justice 29, 36, 40, 42-43, 60,158 youth courts see Tribunales Tutelares de Menores YouTube 18, 200, 203-205 ¿̇Y ahora qué, señor fiscal? 7, 68, 74 